\title{
Factors Associated with Safe Infant Sleep Practices in Saudi Arabia
}

\author{
Hassan Al-shehri (D) \\ Rahaf Almozaai ${ }^{2}$ \\ Marwh Kariri ${ }^{2}$ \\ Yara Alhazmi $\mathbb{D}^{2}$ \\ Shatha AIDakhel ${ }^{2}$ \\ Reyouf Alhunaishel ${ }^{2}$ \\ Dina Aladhadhi ${ }^{2}$ \\ 'Department of Pediatrics, College of \\ Medicine, Imam Mohammad Ibn Saud \\ Islamic University, Riyadh, Saudi Arabia; \\ ${ }^{2}$ College of Medicine, Imam Mohammad \\ Ibn Saud Islamic University, Riyadh, Saudi \\ Arabia
}

Correspondence: Hassan Al-shehri Department of Pediatrics, College of Medicine, Imam Mohammad Ibn Saud Islamic University, Riyadh, Saudi Arabia Tel +966II2582759

Email haalshehri@imamu.edu.sa
Purpose: To assess mothers' approaches to infant sleep practices.

Patients and Methods: A cross-sectional survey study was conducted on mothers with babies aged below five months who were attending paediatric clinics between November 1st, 2020, and January 5th, 2021, in Riyadh, Saudi Arabia.

Results: A total of 522 mothers participated in this study. A total of $38.9 \%$ practised exclusive formula feeding. A total of $61.9 \%$ of the participants practised the supine position. The majority $(93.3 \%)$ of the mothers shared a room with their babies, while $34.7 \%$ shared a bed. Only $6.9 \%$ did not use any soft bedding. Age was a significant predictor associated with participant practices regarding sleeping and feeding positions $(\mathrm{p}<0.05)$. Having two or more children was associated with improper sleeping practices $(\mathrm{p}<0.05)$. Being non-Saudi and having a university degree or higher were associated with having a higher risk of unsafe practices regarding bed-sharing $(\mathrm{p}<0.05)$. On the other hand, being contacted by a doctor, nurse, or other healthcare worker about safe sleep practices were an important factor that influenced safe practices regarding feeding $(\mathrm{p}<0.05)$. Receiving care at a private hospital was associated with safer practices regarding sleeping position and bed-sharing $(p<0.05)$.

Conclusion: We observed high-risk sleeping practices among Saudi mothers. This includes using soft bedding and unsafe sleeping positions. The importance of this study lies in the future implementation of this result through public health measures aimed at at-risk populations.

Keywords: practices, Saudi Arabia, sleep, sudden infant death syndrome

\section{Introduction}

The sleep position and surrounding environment of the infant have been controversial internationally because of concerns regarding infant deaths. Despite this, parents in different cultures sleep close to their infants. WEIRD (western, educated, industrial, rich and democratic) settings emphasise separation and minimal bodily contact, particularly at night. ${ }^{1-4}$ At the same time, it is important to note that these models are incongruent with maternal-infant evolved biology, and differ from infant care in other societies and cultures where a vast majority of the global population resides. $^{5-7}$ Three decades ago saw the beginning of engagement by anthropologists with infant sleep. Solitary infant sleep practices were dominant in the anthropologists' own societies and they used this practice to judge other cultures even though this was considered to be an unusual and historically recent behaviour confined to a limited sub-group of cultures. ${ }^{1}$ Based on these perceptions, the anthropologists explored the potential negative relationship between solitary infant sleep arrangements and sudden and unexpected infant death. ${ }^{8,9}$ 
The issue of babies sleeping with their parents has been a polarising topic due to different theoretical and ideological perspectives, and contradictory evidence. ${ }^{5}$ From an evolutionary perspective, night-time infant care through close mother-infant sleep proximity (co-sleeping, initially defined as mother and baby sharing a bed) might reduce sudden infant deaths (SIDS) ${ }^{8}$ as infants undergo physiological changes in their breathing control around three months of age that increase their vulnerability to unpredictable breathing cessation. ${ }^{10}$

Co-sleeping was redefined to encompass roomsharing, ${ }^{10}$ while bed-sharing was defined to include all shared sleeping surfaces (beds, armchairs, sofas, and makeshift arrangements). Bed-sharing appeared to be a dangerous activity, as many babies died in bed-sharing scenarios. Strict guidance was introduced to prevent parents from bed-sharing in many countries, including the United States and United Kingdom. ${ }^{11}$

Several factors associated with unsafe sleep practices and sleep environments have been shown to pose a higher risk of SIDS. The sudden death of infants under the age of one year remains unexplained despite thorough case investigations, including the performance of a complete autopsy, examination of the death scene, and a review of the clinical history. ${ }^{12}$ There have been few studies that have explored SIDS in Saudi Arabia. A previous study estimated that the incidence rate of SIDS is around 1.3 per 1000 live births. ${ }^{13}$ All the deaths in this study were certified by a medical doctor and the diagnosis was made by excluding known causes of death. Child health organisations have made specific recommendations for caregivers to promote safe sleep practices and reduce the risk of sudden infant death. To begin with, the supine position, whereby an infant sleeps wholly on its back, is appropriate and safe. The prone position (on the stomach) or side sleeping, whereby they can easily roll into the prone position, are to be avoided. Second, infants should be placed on a firm sleep surface, for example, a mattress in a safetyapproved cot covered by fitted bedclothes with no other blankets, pillows, toys, or soft objects near to them. This is because such objects can obstruct an infant's nose and mouth, possibly leading to suffocation or SIDS. Additionally, soft surfaces, such as sofas or couches, should be avoided. Third, it is recommended for infants to sleep in their parental bedroom, close to the parents' bed, to make them comfortable and allow for feeding, but they should be on a separate sleep surface (in their crib) (room-sharing is recommended, while bed-sharing is discouraged). Additionally, the American Academy of Pediatrics (AAP) has recommended the following infant sleep safety practices for babies up to one year of age: babies should sleep on their backs for all sleep times - for naps and at night - using a firm sleep surface; the baby should only be brought into your bed to feed or comfort it; bed-sharing is not recommended for any babies; alternatively, a baby's sleep area should be set up in the same room for the first six months or, ideally, for the first year; soft objects, loose bedding, or any objects that could increase the risk of entrapment, suffocation, or strangulation should be kept out of the baby's sleep area; the child should not be allowed to fall asleep on nursing pillows or pillow-like lounging pads; the child should never be placed to sleep on a couch, sofa, or armchair, and a pacifier should be given to babies at nap time and bedtime when they are being breastfed. ${ }^{14}$

Mothers should avoid active or passive smoking during pregnancy, and even after the infant's birth. Measures should be taken to prevent overheating, and covering infants' faces and heads should be avoided. ${ }^{15,16}$ Substantial epidemiological evidence suggests that there is a lower risk of SIDS among infants whose mothers have obtained regular prenatal care. ${ }^{17,18}$ Therefore, pregnant women should follow guidelines regarding the frequency of prenatal visits. ${ }^{19}$ Also, they should be encouraged to breastfeed and to avoid bottles or formula because breastfeeding reduces the risk of SIDS and offers other health benefits. ${ }^{15}$ Studies have found that infants born preterm or who have low birth weight are at high risk of SIDS compared with infants born at term. The risk of SIDS increases as birth weight or gestation decreases. ${ }^{20,21}$ According to the recommendations of the AAP and the Centers for Disease Control and Prevention (CDC), infants should be immunised as evidence suggests that vaccination may have a protective effect against SIDS. ${ }^{22,23}$ There are limited studies on safe sleep practices in Saudi Arabia. ${ }^{24-27}$ Therefore, the purpose of this study is to assess infant sleeping practices conducted by mothers in Saudi Arabia. Additionally, we aimed to determine the prevalence of unsafe infant sleep practices and their associated predictors.

\section{Materials and Methods Study Design}

This was a prospective cross-sectional survey study that was carried out between November 1st, 2020, and 
January 5th, 2021, in paediatric clinics in government and private hospitals in Riyadh, Saudi Arabia.

\section{Study Population}

The study population was recruited from mothers with babies aged below five months who were attending paediatric clinics in six government healthcare centres (1- Imam Mohammad Ibn Saud Islamic University Medical Centre, 2King Saud Medical City, 3- King Fahad Medical City, 4 King Abdullah Specialist Children Hospital, 5- the Security Forces Hospital, and 6- the Primary Healthcare Centre-Al Hamra District) and two private healthcare centres (1Dr Sulaiman Al-Habib Medical Services Group, and 2- Al Mouwasat Hospital) in Riyadh, Saudi Arabia.

This age range was chosen because the incidence of SIDS is highest between one and four months of age. Mothers who had children with congenital deformities and mothers who had had their babies admitted to the neonatal intensive care unit (NICU) were excluded. Babies with congenital deformities and who receive intensive care will have other health issues that may interfere with their health status and affect their sleeping patterns, thus requiring special arrangements and sleeping environments, unlike normal healthy babies. At the paediatric clinics, the researcher invited the mothers to participate in the study after explaining the study's aim and objectives to them. If they agreed, they were asked to complete a questionnaire after signing the participant consent form. Once the mothers had answered all the questions, they were given an awareness brochure about safe sleeping positions for infants, Appendix 1.

\section{Sample Size}

To power the study, using a standard deviation of 0.5 and a margin of error of $5 \%$, a minimum targeted sample size of 385 women was required to have a $95 \%$ chance of detecting a difference.

\section{The Questionnaire}

The study questionnaire was developed based on the recommendations of the AAP regarding safe infant sleep practices. ${ }^{28,29}$ The questionnaire included 12 questions covering demographic data, infant sleep and care practices, and the sociodemographic characteristics of the target population, Appendix 2. The sociodemographic data included maternal age, education, antenatal smoking patterns, other household smokers, and the number of children. The questions on infant care practices asked about the usual place of sleep, bedsharing, soft bedding, and types of feeding. We focused on the features of high-risk mothers that are potentially associated with SIDS, including smoking during pregnancy and whether there were smokers in the infant's environment after birth, young maternal age, late or no prenatal care, poor education, and feeding.

\section{Ethics}

Ethical approval for the study was obtained from the Imam Mohammad Ibn Saud Islamic University Institutional Review Board (project number: 85-2020). Informed consent was obtained from the mothers before they participated in the study. This study was conducted in accordance with the Declaration of Helsinki.

\section{Statistical Analysis}

Data were analysed using Statistical Package for Social Scientists version 26.0. Continuous data were reported as mean ( \pm standard deviation [SD]). Categorical data were reported as frequency (percentage). The respondents were categorised into groups based on variables such as safe and unsafe feeding practices, infant sleeping position, and bed-sharing. Unsafe feeding practices were used as the point of reference during the study. Binary logistic regression analysis was used to analyse the association between safe and unsafe maternal practices and demographic factors such as education, level of income, and maternal age. A p-value of less than 0.5 was considered statistically significant, with a confidence interval of $95 \%$.

\section{Results}

\section{Characteristics of the Study Participants}

A total of 522 mothers participated in this study. More than half of them $(53.4 \%)$ were aged $26-35$ years. Just under half, $49.0 \%$, of them had two or more children, while $24.1 \%$ had no other children. Most of them (93.1\%) were Saudis. Fewer than half of them (40.2\%) had previously discussed safe practices with a doctor, a nurse, or other healthcare practitioners, see Table 1.

\section{Safe Infant Sleep Practices}

Table 2 below shows infant sleep practices among the participating mothers. Only $38.9 \%$ of the study participants practised exclusive formula feeding, with $61.1 \%$ of them practising other types of feeding (combined and formula feeding). Regarding the usual sleeping position, 
Table I Demographic Characteristics of the Study Participants $(n=522)$

\begin{tabular}{|c|c|c|}
\hline Demographic Variable & Frequency & (\%) \\
\hline \multicolumn{3}{|l|}{ Maternal age (years): } \\
\hline Less than 20 years & 11 & $2.1 \%$ \\
\hline $20-25$ years & 120 & $23.0 \%$ \\
\hline $26-35$ years & 279 & $53.4 \%$ \\
\hline $36-40$ years & 94 & $18.0 \%$ \\
\hline 40 years and above & 18 & $3.5 \%$ \\
\hline \multicolumn{3}{|c|}{ Number of children (other than the current newborn) } \\
\hline None & 126 & $24.1 \%$ \\
\hline One & 139 & $26.6 \%$ \\
\hline Two or more & 257 & $49.2 \%$ \\
\hline \multicolumn{3}{|l|}{ Nationality } \\
\hline Saudi & 486 & $93.1 \%$ \\
\hline Non-Saudi & 36 & $6.90 \%$ \\
\hline \multicolumn{3}{|l|}{ Level of Education } \\
\hline Primary education & 41 & $7.9 \%$ \\
\hline High school level & 147 & $28.2 \%$ \\
\hline University degree or higher & 334 & $64.0 \%$ \\
\hline \multicolumn{3}{|c|}{$\begin{array}{l}\text { Did a doctor, nurse, or other healthcare worker talk with you } \\
\text { about the safe practices for your baby? }\end{array}$} \\
\hline Yes & 210 & $40.2 \%$ \\
\hline \multicolumn{3}{|l|}{ Hospital type } \\
\hline Government & 239 & $45.8 \%$ \\
\hline Private & 283 & $54.2 \%$ \\
\hline
\end{tabular}

the study showed that only $61.9 \%$ of the participants practised the supine position, and prone position and the side position were practised by $11.5 \%$ and $26.6 \%$ of them, respectively. Concerning sleeping habits, $93.3 \%$ of the mothers shared a room with their babies, while one-third (34.7\%) of the study participants reported that they shared the bed with their baby. When asked about social habits, $4.79 \%$ of the mothers reported smoking either during antenatal or post-natal care (maternal smoking), while $36.6 \%$ reported having at least one smoker at home.

Regarding soft bedding, most of the participating women $(83.3 \%)$ used duvets, while $6.9 \%$ did not use any soft bedding. The proportion of mothers who carried out
Table 2 Safe Infant Sleep Practices Among Mothers (N= 522)

\begin{tabular}{|c|c|c|}
\hline Safe Infant Sleep Practices & Frequency & (\%) \\
\hline \multicolumn{3}{|c|}{ What is the type of feeding you are providing for your baby } \\
\hline Exclusive breast feeding & 135 & $25.9 \%$ \\
\hline Combined & 184 & $35.2 \%$ \\
\hline Formula feeding & 203 & $38.9 \%$ \\
\hline \multicolumn{3}{|c|}{ What is the usual sleeping position for your baby? } \\
\hline Supine & 323 & $61.9 \%$ \\
\hline Side & 139 & $26.6 \%$ \\
\hline Prone & 60 & $11.5 \%$ \\
\hline
\end{tabular}

Have you ever been smoker (either antenatal or post-natal)?

\begin{tabular}{|l|c|c|}
\hline Yes & 25 & $4.8 \%$ \\
\hline
\end{tabular}

Is there any one smoker at home? (Household smoking)

\begin{tabular}{|c|c|c|}
\hline Yes & 191 & $36.6 \%$ \\
\hline
\end{tabular}

Do you usually sleep with your baby in the same room?

\begin{tabular}{|l|l|l|}
\hline Yes & 487 & $93.3 \%$ \\
\hline
\end{tabular}

Does your baby usually sleep with you on the same bed?

\begin{tabular}{|c|c|c|}
\hline Yes & 181 & $34.7 \%$ \\
\hline \multicolumn{2}{|c|}{ Do you use any of soft bedding methods in your baby bed? } \\
\hline Yes & 486 & $93.1 \%$ \\
\hline \multicolumn{3}{|c|}{ Soft bedding methods (more than one answer could be chosen) } \\
\hline Duvet & 435 & $83.3 \%$ \\
\hline Pillow & 413 & $79.1 \%$ \\
\hline Cot bumper & 322 & $61.7 \%$ \\
\hline
\end{tabular}

safe infant sleep practices is highlighted in Figure 1. Around one-quarter $(25.9 \%)$ of the participating women carried out safe feeding practices with their infants. Around two-thirds $(61.9 \%)$ of the participating women used safe sleeping positions, with a similar proportion $(65.3 \%)$ committed to safe bed-sharing practices.

\section{Predictors of Safe Sleep Practices}

Logistic regression was performed to determine the association between the demographic factors (independent variables) with feeding, sleeping position, bed-sharing and soft bedding (dependent variables), as shown in Table 3. The study results showed that age was 


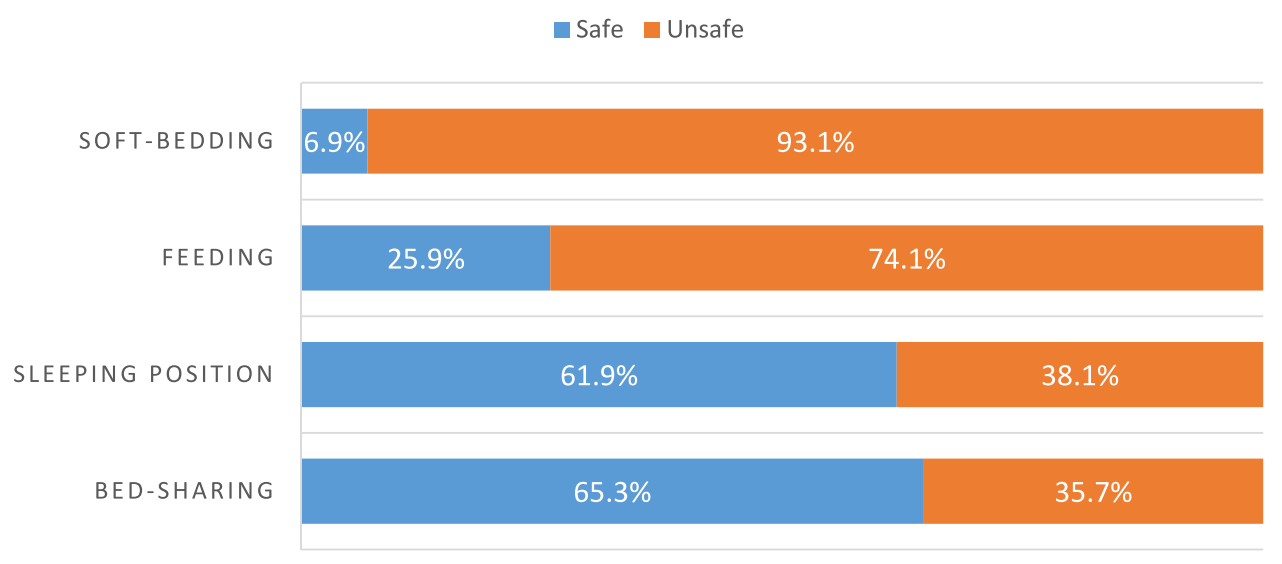

Figure I Safety of infant sleep practices among study participants.

a significant predictor that affected the participants' practices regarding sleeping and feeding positions $(p<0.05)$. Having two or more children was an important variable that was associated with negative sleeping practices $(\mathrm{p}<0.05)$. Being non-Saudi and having a university degree or higher were associated with unsafe practices regarding bed-sharing $(\mathrm{p}<0.05)$. On the other hand, being contacted by a doctor, nurse, or other healthcare worker about safe sleep practices were an important factor that influenced safe practices regarding feeding $(\mathrm{p}<0.05)$. Receiving care at a private hospital was associated with safer practices regarding sleeping position and bed-sharing $(p<0.05)$.

\section{Discussion}

There are serious concerns about infant sleep practices carried out by mothers, especially with the high risk of SIDS or asphyxia due to poor positioning during sleep. Access to relevant knowledge and information plays a key role in the adoption of safe sleeping practices, especially in countries such as Saudi Arabia, where education opportunities for women are still evolving. Studying routine practices used by mothers regarding infant sleeping positions is necessary for the identification of potential risks and the quality of the child's sleep, which contributes significantly to their growth and development. This study provides important insights into the development of relevant frameworks to promote the adoption of safe infant sleep practices by mothers to reduce the incidence of sleep-related complications through assessing infant sleep practices by mothers in Riyadh, Saudi Arabia.

By employing a variety of sleeping practices, we found that fewer than half of the respondents $(40.2 \%)$ received medical advice that was in line with the recommendations of the AAP. The AAP states that to reduce the risk of SIDS and suffocation, infants should be placed in a supine position on a firm, flat sleep surface covered by a fitted sheet, with no other bedding or soft objects. Infants should never be left to sleep on sofas or on any furniture used for sitting. Sharing a room but not a bed, not smoking during pregnancy and after delivery, seeking regular prenatal care, and breastfeeding are encouraged. ${ }^{28}$

Mothers who had received information about safe infant sleep practices from healthcare practitioners (HCP) were more likely to report them than those who had not. Regarding the usual sleeping position, most of the respondents $(61.9 \%)$ placed their infants in the supine position, $11.5 \%$ placed them in the prone position, and $26.6 \%$ of the mothers placed their infants in the side sleeping position. A similar study, which was conducted in Saudi Arabia at King Abdulaziz University Hospital, found that the usual infant sleep position was a supine position, and none of the infants was found in the prone position. ${ }^{26}$ Conversely, another study conducted in Al Ain city, UAE, showed an even higher prevalence of infants sleeping in a prone position, this being reported by $18 \%$ of respondents. ${ }^{24}$ Similar results were found in another study conducted in Australia, which showed that the supine position was the most reported, with only $2 \%$ of mothers reporting the use of the prone position. ${ }^{25}$

A study of the sleeping habits showed that $93.3 \%$ of the participating mothers slept in the same room as their babies, while only $6.7 \%$ slept in separate rooms. Of the mothers who slept in the same room as their babies, 34.7\% shared a bed while the rest had a separate bed or cot for the baby. Although room-sharing lowers the risk of SIDS, ${ }^{30-32}$ at the same time, parents should be aware not to share their bed with their babies as it is a high-risk practice associated with SIDS. ${ }^{31-33}$ At the same time, 
Table 3 Predictors of Safe Sleep Practices

\begin{tabular}{|c|c|c|c|}
\hline Demographic Variable & $\begin{array}{c}\text { Odds Ratio of Safe } \\
\text { Practices Regarding } \\
\text { Feeding }(95 \% \mathrm{CI})\end{array}$ & $\begin{array}{l}\text { Odds Ratio of Safe Practices } \\
\text { Regarding Sleeping Position } \\
\qquad(95 \% \mathrm{Cl})\end{array}$ & $\begin{array}{l}\text { Odds Ratio of Safe } \\
\text { Practices Regarding Bed- } \\
\text { Sharing }(95 \% \mathrm{Cl})\end{array}$ \\
\hline \multicolumn{4}{|l|}{ Maternal age (years): } \\
\hline Less than 20 years (Reference category) & 1.00 & 1.00 & 1.00 \\
\hline 20-25 years & $1.54(0.98-2.40)$ & $1.95(1.25-3.07)^{* *}$ & $0.98(0.64-1.5 I)$ \\
\hline $26-35$ years & $0.6 \mathrm{I}(0.4 \mathrm{I}-0.9 \mathrm{I})^{*}$ & $1.15(0.81-1.64)$ & $1.22(0.85-1.74)$ \\
\hline $36-40$ years & $1.12(0.68-1.85)$ & $0.58(0.37-0.91)^{*}$ & $0.83(0.52-1.3 I)$ \\
\hline 40 years and above & $3.00(1.17-7.72)^{*}$ & $0.30(0.11-0.80)^{*}$ & $1.06(0.39-2.88)$ \\
\hline \multicolumn{4}{|c|}{ Number of children (other than the current newborn) } \\
\hline None (Reference category) & 1.00 & 1.00 & 1.00 \\
\hline One & $0.86(0.55-1.35)$ & $1.00(0.67-1.49)$ & I.0I (0.67-I.52) \\
\hline Two or more & $1.06(0.72-1.57)$ & $0.68(0.47-0.96)^{*}$ & I.II (0.78-I.59) \\
\hline \multicolumn{4}{|l|}{ Nationality } \\
\hline Saudi (Reference category) & 1.00 & 1.00 & 1.00 \\
\hline Non-Saudi & $2.79(I .4 I-5.54)^{* *}$ & $1.10(0.54-2.22)$ & $0.45(0.23-0.88)^{*}$ \\
\hline \multicolumn{4}{|l|}{ Level of Education } \\
\hline Primary education (Reference category) & 1.00 & 1.00 & 1.00 \\
\hline High school level & $0.78(0.49-1.2 \mathrm{I})$ & $0.93(0.63-1.37)$ & $0.69(0.47-1.02)$ \\
\hline University degree or higher & $1.18(0.78-1.77)$ & $1.21(0.84-1.74)$ & $1.59(1.10-2.31)^{*}$ \\
\hline \multicolumn{4}{|c|}{ Did a doctor, nurse, or other healthcare worker talk with you about the safe practices for your baby? } \\
\hline No (Reference category) & 1.00 & 1.00 & 1.00 \\
\hline Yes & $1.98(1.33-2.94)^{* *}$ & $1.19(0.83-1.71)$ & $1.32(0.91-1.92)$ \\
\hline \multicolumn{4}{|l|}{ Hospital type } \\
\hline Government (Reference category) & 1.00 & 1.00 & 1.00 \\
\hline Private & $1.27(0.86-1.88)$ & $1.86(1.30-2.66)^{* *}$ & $1.51(1.05-2.17)^{*}$ \\
\hline
\end{tabular}

Notes: ${ }^{*}<0.05 ;{ }^{* *} p<0.01$.

unplanned sleeping or sleeping with babies in unsafe places such as sofas or couches are associated with a high risk of SIDS. ${ }^{30,31}$

The type of bedding on which babies sleep is also considered a contributing factor for sudden infant death. Our study showed that $83.3 \%$ used duvets, $79.1 \%$ used pillows, and $61.7 \%$ used cot bumpers for their babies, while only $6.9 \%$ of mothers did not use any soft bedding. These are considered to be alarming findings as most of the participants reported unsafe practices concerning using these soft bedding methods. Using soft bedding, duvets, bumpers, and pillows are associated with higher risk of covering the baby's head and, therefore, increase the risk of SIDS. ${ }^{30,34,35}$ Infants who sleep with soft bedding have a five-fold increased risk of sudden infant mortality regardless of sleep position, and a 21-fold increased risk if they sleep prone. ${ }^{30}$ According to AAP guidelines, infants should be put on a firm, flat sleep surface covered by a fitted sheet with no other bedding or soft objects to reduce the risk of SIDS and suffocation. Infants should never be left to sleep on sofas or on any furniture designed for sitting. ${ }^{36}$ Recliners, cushioned chairs, and makeshift 
beds are also dangerous, placing infants at higher risk for suffocation or asphyxia. ${ }^{37}$

Investigation into their social habits revealed that $4.8 \%$ smoked either during antenatal or post-natal care, while $36.6 \%$ were found to have at least one smoker at home. Maternal smoking is positively correlated with worsening arousal processes, which increase the risks of SIDS. ${ }^{38-40}$ Smoker pregnant women should be informed that both prenatal and post-natal (through passive smoking) smoking are high-risk factors for SIDS. ${ }^{37}$ Additionally, they should be advised to stop smoking even before planning to be pregnant. ${ }^{35,37}$ Smoking has lately been recognised as the most important current modifiable risk factor in reducing the risk of SIDS. Furthermore, infants exposed to smoke during pregnancy are more likely to be born preterm and with low birth weight. ${ }^{41}$ Antenatal or post-natal smoking was reported by the participants to be $4.79 \%$, while $36.6 \%$ of the mothers were found to have at least one smoker at home.

Investigation into the infant feeding practices revealed that only $25.9 \%$ of the mothers practised exclusive breastfeeding, 35.2\% used combined breastfeeding and baby formula, and 38.9\% used formula feeding only. Breastfeeding creates a strong emotional bond between the mother and her child, which is known to result in emotional stability and healthy psychological development and subsequent behavioural aspects. Breastfeeding has numerous multidimensional benefits for the baby and the mother. These include socioeconomic, nutritional, environmental, and psychological beneficial outcomes. ${ }^{42,43}$ It is, therefore, recommended that breastfeeding be continued exclusively for at least six months, and to continue as long as the baby is accepting it and the mother is able to offer it. ${ }^{42,43}$

Concerning the influence of age on the adoption of sleeping practices by mothers, the study findings showed that those aged 36 years and above have a higher likelihood of practising unsafe sleeping positions compared to others. This could be justified as younger mothers might have increased access to maternal health education through schooling, interactions with HCPs, or social media. On the relationship between sleeping position and the use of cot bumpers, the highest number of mothers who placed their infants in the supine position also used cot bumpers, while those who placed their infants in the prone position reported the least use of cot bumpers. Moreover, a significant positive correlation was found between the preferences for combined feeding practices in mothers with two or more children compared to those with no other children. Mothers who went to government hospitals have a higher likelihood of adopting combined infant feeding and unsafe sleeping practices compared to those who went to private hospitals.

Regarding sleeping positions, results regarding prone relative to supine, given the other variables in the model, were constant; mothers using governmental hospitals were more likely to prefer prone sleeping positions than mothers using private hospitals. This may indicate a serious issue as sleeping in a prone position is associated with an increased risk of respiratory compromise and, subsequently, SIDS. Concerning the preferred infant sleeping position and the mother's age, older mothers were more likely than younger mothers to prefer the side sleeping position to supine. In another study conducted in Jordan, most mothers preferred the side position over the supine position. ${ }^{44}$ It is worth mentioning that parents' knowledge, attitudes, and practices concerning their children are affected by different variables, and this is ultimately reflected in their different approaches to raising and interacting with their babies. ${ }^{45}$ Parents knowledge about safe sleep environments is critical for their development. $^{45}$ Infant sleep awareness and practices are highly influenced by the parents' culture, social ideology, and demographic disparities. ${ }^{46-49}$ Changing these influencing factors is not easy and healthcare professionals need to start educating parents on proper infant sleep practices early, even before they plan to be pregnant. ${ }^{46}$

Safe and healthy infant sleeping practices should be considered to be a major constituent of maternal awareness programmes. Healthcare practitioners should provide proper counselling about the correct practices regarding infants' health, and there should be community campaigns to address the current issues. Health education campaigns are recommended to increase awareness of safe sleep practices among the general population to encourage the supine sleeping position and to discourage other risky practices. This should result in a significant reduction in the incidence of SIDS. These campaigns should target high-risk populations, such as older mothers (aged 36 years and above), those who have two or more children, and Saudis.

\section{Study Limitation}

The use of a convenience sampling technique could have affected the socioeconomic and demographic characteristics of the study participants and limited the generalizability of our findings. The questionnaire asked only for the usual place of sleep and did not investigate practices related to unplanned sleeping, knowing that many deaths occur when 
sleep is unplanned on an unsafe surface (such as a couch and sofa). A self-reported questionnaire does not necessarily describe the actual behaviour of the study participants. The dichotomy of safe and unsafe practices in this paper is too simplistic and the study does not explore infant sleep environment in the detail required to contribute to improving infant safety. Our study did not ask about preterm or low birth weight infants, which could be an important factor that might affect their safe sleep practices. Therefore, the findings should be interpreted with care.

\section{Conclusion}

The majority of the mothers in our study population used the supine sleep position and the majority of infants were exposed to other care practices that have been linked to SIDS. More than half of the mothers did not receive any information from their HCP. As there are no other similar published studies on infant sleeping practices in Saudi Arabia, our findings provide the base for the elaboration of health education campaigns. Future studies should explore detailed sleep practices to identify all risky practices and provide suitable educational intervention to minimise the risk of SIDS.

\section{Disclosure}

The authors declare no conflicts of interest in this work.

\section{References}

1. Trevathan WR, Rosenberg K. Costly and Cute: Helpless Infants and Human Evolution. University of New Mexico Press; 2017.

2. Jones CHD, Ball HL. In sleep: multi-professional perspectives. In: Medical Anthropology and Children's Sleep. London: Jessica Kingsley Publishers; 2012:86-103.

3. Ball HL, Russell CK. In evolution, early experience and human development: from research to practice and policy. In: Narvaez D, Schore A, Gleason T, editors. Nighttime Nurturing: An Evolutionary Perspective on Breastfeeding and Sleep. Oxford University Press; 2012:241-261.

4. Ball HL. Medicine and evolution: current applications, future prospects. In: Elton S, O'Higgins P, editors. Evolutionary Paediatrics: A Case Study in Applying Darwinian Medicine. London: Taylor \& Francis; 2008:125-150.

5. Ball HL. The Atlantic divide: contrasting U.K. and U.S. recommendations on cosleeping and bed-sharing. J Hum Lact. 2017;33(4):765-769. doi:10.1177/0890334417713943

6. McKenna JJ, Ball HL, Gettler LT. Mother-infant cosleeping, breastfeeding and sudden infant death syndrome: what biological anthropology has discovered about normal infant sleep and pediatric sleep medicine. Am J Phys Anthropol. 2007;134(S45):133-161. doi:10. 1002/ajpa.20736

7. Airhihenbuwa CO, Iwelunmor JI, Ezepue CJ, Williams NJ, JeanLouis G. I sleep, because we sleep: a synthesis on the role of culture in sleep behavior research. Sleep Med. 2016;18:67-73. doi:10.1016/j. sleep.2015.07.020
8. McKenna JJ. An anthropological perspective on the sudden infant death syndrome (SIDS): the role of parental breathing cues and speech breathing adaptations. Med Anthropol. 1986;10(1):9-92. doi:10.1080/01459740.1986.9965947

9. Konner M, Super CM. In the role of culture in developmental disorder. In: Super CM, editor. Sudden Infant Death Syndrome: An Anthropological Hypothesis. San Diego: Academic Press; 1987:95-108.

10. McKenna JJ, Mosko S. Evolution and the sudden infant death syndrome (SIDS), part III: infant arousal and parent-infant co-sleeping. Human Nature. 1990;1(2):291-396. doi:10.1007/BF02733987

11. Bartick M. Should the AAP Sleep Alone? 2014.

12. Willinger M, James L, Catz C. Defining the sudden death syndrome (SIDS): deliberations of an expert panel convened by the National Institute of Child Health and Human Development. Pediatric Pathol. 1991;11(5):677-684. doi:10.3109/15513819109065465

13. Srair H, Owa J, Aman H. Cause-specific infant mortality rate in Qatif area, eastern province, Saudi Arabia. Ann Saudi Med. 1995;15:156-158. doi:10.5144/0256-4947.1995.156

14. Moon R. How to keep your sleeping baby safe: AAP policy explained. 2021; Available from: https://www.healthychildren.org/ English/ages-stages/baby/sleep/Pages/A-Parents-Guide-to-SafeSleep.aspx. Accessed November 26, 2021.

15. American Academy of Pediatrics. Task force on infant sleep position and sudden infant death Syndrome. The changing concept of sudden infant death syndrome: diagnostic coding shifts, controversies regarding the sleeping environment, and new variables to consider in reducing risk. Pediatrics. 2005;116(5):1245-1255. doi:10.1542/peds.20 05-1499

16. Horne R, Hauck F, Moon R. Sudden infant death syndrome and advice for safe sleeping. BMJ. 2015;350:1-7. doi:10.1136/bmj.h1989

17. Getahun D, Amre D, Rhoads G, Demissie K. Maternal and obstetric risk factors for sudden infant death syndrome in the United States. Obstet Gynecol. 2004;103(4):646-652. doi:10.1097/01.AOG.000011 7081.50852 .04

18. Stewart A, Williams S, Mitchell E, Taylor B, Ford R, Allen E. Antenatal and intrapartum factors associated with sudden infant death syndrome in the New Zealand Cot Death Study. J Paediatr Child Health. 1995;31(5):473-478. doi:10.1111/j.1440-1754.1995. tb00861.x

19. American Academy of Pediatrics Committee on Fetus and Newborn. ACOG Committee on Obstetric Practice. Guidelines for Perinatal Care. Elk Grove Village, IL: American Academy of Pediatrics; 2012.

20. Mitchell EA, Taylor BJ, Ford RP, et al. Four modifiable and other major risk factors for cot death: the New Zealand study. J Paediatr Child Health. 1992;28(Suppl 1):S3-S8. doi:10.1111/j.1440-1754.19 92.tb02729.x

21. Mitchell EA, Tuohy PG, Brunt JM, et al. Risk factors for sudden infant death syndrome following the prevention campaign in New Zealand: a prospective study. Pediatrics. 1997;100(5):835-840. doi:10.1542/peds.100.5.835

22. Fleming P, Blair P, Platt M, Tripp J, Smith I, Golding J. The UK accelerated immunization program and sudden unexpected death in infancy: case- control study. BMJ. 2001;322(7290):1-5. doi:10.1136/ bmj.322.7290.822

23. Mitchell E, Stewart A, Clements M, Ford R. New Zealand Cot Death Study Group. Immunisation and the sudden infant death syndrome. Arch Dis Child. 1995;73(6):498-501. doi:10.1136/adc.73.6.498

24. Abdulrazzaq YM, Kendi AA, Nagelkerke N. Child care practice in the United Arab Emirates: the ESACCIPS study. Acta paediatrica. 2008;97(5):590-595. doi:10.1111/j.1651-2227.2008.00758.x

25. Cole R, Young J, Earney L, Kearney L, Thompson J. Awareness of infant safe sleep messages and associated care practices: findings from an Australian cohort of families with young infants. $B M J$ Paediatrics Open. 2021;5(1):e000972. doi:10.1136/bmjpo-2020000972 
26. Sobaihi M, Banjari MA, Alahmadi TS. Implementation of safe sleep practice recommendations for infants in inpatient wards. Cureus. 2020;12(10):e11155. doi:10.7759/cureus.11155

27. Alahmadi T, Sobaihi M, Banjari M, Bakheet K, Modan Alghamdi S, Alharbi A. Are safe sleep practice recommendations for infants being applied among caregivers? Cureus. 2020;12(12):e12133. doi:10. 7759/cureus. 12133

28. American Academy of Pediatrics. Safe sleep and your baby: how parents can reduce the risk of SIDS and suffocation - 50/pk [Brochure]. 2016; Available from: https://shop.aap.org/safe-sleepand-your-baby-how-parents-can-reduce-the-risk-of-sids-andsuffocation-brochure/. Accessed August 10, 2021.

29. Moon RTFOSIDS. SIDS and other sleep-related infant deaths: evidence base for 2016 updated recommendations for a safe infant sleeping environment. Pediatrics. 2016;138(5):e20162940. doi:10. 1542/peds.2016-2940

30. Hauck F, Herman S, Donovan M, et al. Sleep environment and the risk of sudden infant death syndrome in an urban population: the Chicago infant mortality study. Pediatrics. 2003;111(5 Pt 2):1207-1214. doi:10.1542/peds.111.S1.1207

31. Blair P, Fleming P, Smith I, et al. Babies sleeping with parents: case-control study of factors influencing the risk of the sudden infant death syndrome. BMJ. 1999;319:1457-1462. doi:10.1136/bmj.31 9.7223.1457

32. Carpenter R, Irgens L, Blair P, et al. Sudden unexplained infant death in 20 regions in Europe: case control study. Lancet. 2004;363:185-191. doi:10.1016/S0140-6736(03)15323-8

33. Scragg R, Mitchell E, Taylor B, et al. Bedsharing, smoking and alcohol in the sudden infant death syndrome. BMJ. 1993;207:1312-1318. doi:10.1136/bmj.307.6915.1312

34. McGarvey C, McDonnell M, Chong A, O’Regan M, Matthews T. Factors relating to the infant's last sleep environment in sudden infant death syndrome in the Republic of Ireland. Arch Dis Child. 2003;88:1058-1106. doi:10.1136/adc.88.12.1058

35. Moon R, Mathews A, Joyner B, Oden R, He J, McCarter MR. Health messaging and African-American infant sleep location: a randomized controlled trial. J Community Health. 2017;42(1):1-9. doi:10.1007/ s10900-016-0227-1

36. American Academy of Pediatrics. Safe sleep recommendations. 2021; Available from: https://www.aap.org/en-us/advocacy-andpolicy/aap-health-initiatives/safe-sleep/Pages/Safe-SleepRecommendations.aspx. Accessed August 10, 2021.

37. Canadian Paediatric Society. Recommendations for safe sleeping environments for infants and children. Paediatr Child Health. 2004;9(9):659-672. doi:10.1093/pch/9.9.659
38. Chong D, Yip P, Karlberg J. Maternal smoking: an increasing unique risk factor for sudden infant death syndrome in Sweden. Acta paediatrica. 2004;93(4):471-478. doi:10.1080/080352503 10023495

39. MacDorman M, Cnattingius S, Hoffman H, Kramer M, Haglund B. Sudden infant death syndrome and smoking in the United States and Sweden. Am J Epidemiol. 1997;146(3):249-257. doi:10.1093/oxfordjournals.aje.a009260

40. Malloy M, Hoffman H, Peterson D. Sudden infant death syndrome and maternal smoking. Am J Public Health. 1992;82(10):1380-1382. doi:10.2105/AJPH.82.10.1380

41. Mitchell E, Freemantle J, Young J, Byard R. Scientific consensus forum to review the evidence underpinning the recommendations of the Australian SIDS and kids safe sleeping health promotion programme. J Paediatr Child Health. 2012;48(8):626-633. doi:10. 1111/j.1440-1754.2011.02215.x

42. Shamir R. The benefits of breast feeding. Nestle Nutr Inst Workshop Ser. 2016;86:67-76.

43. Gertosio C, Meazza C, Pagani S, Bozzola M. Breastfeeding and its gamut of benefits. Minerva Pediatr. 2016;68(3):201-212.

44. Hamadneh S, Kassab M, Hamadneh J, Amarin Z. Sudden unexpected infant death in Jordan and the home environment. Pediatr Int. 2016;58(12):1333-1336. doi:10.1111/ped.13016

45. National Academies of Sciences, Engineering, and Medicine. Parenting Matters: Supporting Parents of Children Ages 0-8. Washington (DC): National Academies Press (US); 2016.

46. Chung-Park M. Knowledge, opinions, and practices of infant sleep position among parents. Mil Med. 2012;177(2):235-239. doi:10.72 05/MILMED-D-11-00323

47. Colson E, Rybin D, Smith L, Colton T, Lisler G, Corwin M. Trends and factors associated with infant sleeping position: the national infant sleep position study, 1993-2007. Arch Pediatr Adolesc Med. 2009;163(12):1122-1128. doi:10.10 01/archpediatrics.2009.234

48. Stastny P, Ichinose T, Thayer S, Olson RJ, Keens T. Infant sleep positioning by nursery staff and mothers in newborn hospital nurseries. Nurs Res. 2004;53(2):122-129. doi:10.1097/00006199. 200403000-00008

49. Von Kohorn I, Corwin M, Rybin D, Heeren T, Lister G, Colson E. Influence of prior advice and beliefs of mothers on infant sleep position. Arch Pediatr Adolesc Med. 2010;164(4):363-369. doi:10. 1001/archpediatrics.2010.26
Pediatric Health, Medicine and Therapeutics

\section{Publish your work in this journal}

Pediatric Health, Medicine and Therapeutics is an international, peerreviewed, open access journal publishing original research, reports, editorials, reviews and commentaries. All aspects of health maintenance, preventative measures and disease treatment interventions are addressed within the journal. Practitioners from all disciplines are invited to submit their work as well as healthcare researchers and patient support groups. The manuscript management system is completely online and includes a very quick and fair peer-review system. Visit http://www.dovepress.com/testimonials.php to read real quotes from published authors. 